\title{
BMJ Open Trend analysis of tuberculosis case notifications with scale-up of antiretroviral therapy and roll-out of isoniazid preventive therapy in Zimbabwe, 2000-2018
}

Kudakwashe C Takarinda (D) ,1,2 Anthony D Harries, ${ }^{1,3}$ Tsitsi Mutasa-Apollo, $^{2}$ Charles Sandy, ${ }^{2}$ Regis C Choto, ${ }^{2}$ Simbarashe Mabaya, ${ }^{4}$ Cephas Mbito, ${ }^{2}$ Collins Timire ${ }^{1,2}$

To cite: Takarinda KC, Harries AD, Mutasa-Apollo T, et al. Trend analysis of tuberculosis case notifications with scale-up of antiretroviral therapy and roll-out of isoniazid preventive therapy in Zimbabwe, 2000-2018. BMJ Open 2020;10:e034721. doi:10.1136/ bmjopen-2019-034721

- Prepublication history for this paper is available online. To view these files, please visit the journal online (http://dx.doi. org/10.1136/bmjopen-2019034721).

Received 11 October 2019 Revised 12 February 2020 Accepted 20 March 2020

Check for updates

(C) Author(s) (or their employer(s)) 2020. Re-use permitted under CC BY-NC. No commercial re-use. See rights and permissions. Published by BMJ.

For numbered affiliations see end of article.

Correspondence to Dr Kudakwashe C Takarinda; kctakarinda@gmail.com

\section{ABSTRACT}

Objectives Antiretroviral therapy (ART) and isoniazid preventive therapy (IPT) are known to have a tuberculosis (TB) protective effect at the individual level among people living with HIV (PLHIV). In Zimbabwe where TB is driven by HIV infection, we have assessed whether there is a population-level association between IPT and ART scale-up and annual TB case notification rates (CNRs) from 2000 to 2018.

Design Ecological study using aggregate national data. Setting Annual aggregate national data on TB case notification rates (stratified by TB category and type of disease), numbers (and proportions) of PLHIV in ART care and of these, numbers (and proportions) ever commenced on IPT.

Results ART coverage in the public sector increased from $<1 \%$ (8400 PLHIV) in 2004 to 88\% (>1.1 million PLHIV patients) by December 2018, while IPT coverage among PLHIV in ART care increased from <1\% (98 PLHIV) in 2012 to 33\% (373 917 PLHIV) by December 2018. These HIV-related interventions were associated with significant declines in TB CNRs: between the highest CNR prior to national roll-out of ART (in 2004) to the lowest recorded CNR after national IPT roll-out from 2012, these were (1) for all TB case (510 to 173 cases/100 000 population; $66 \%$ decline, $p<0.001$ ); (2) for those with new TB (501 to 159 cases $/ 100000$ population; $68 \%$ decline, $p<0.001$ ) and (3) for those with new clinically diagnosed PTB (284 to 63 cases $/ 100000$ population; $77.8 \%$ decline, $p<0.001$ ). Conclusions This study shows the population-level impact of the continued scale-up of ART among PLHIV and the national roll-out of IPT among those in ART care in reducing TB, particularly clinically diagnosed TB which is largely associated with HIV. There are further opportunities for continued mitigation of TB with increasing coverage of ART and in particular IPT which still has a low coverage.

\section{BACKGROUND}

Tuberculosis (TB), one of the oldest bacterial infections known to mankind and the HIV infection are a deadly combination. While
Strengths and limitations of this study

- Use of national aggregate data gives a comprehensive picture of the country.

- The ecological design of the study may result in 'ecological fallacy'.

- Despite use of aggregate data, findings are similar to studies using individual data.

- Use of tuberculosis (TB) case notifications does not account for undiagnosed TB cases.

- All factors contributing to declining TB case notifications are not accounted for.

the lifetime risk of developing active ТВ disease among people with latent TB infection (LTBI) is $5 \%-15 \%,{ }^{1}$ those with HIV have a $5 \%-15 \%$ annual risk of acquiring active TB disease. $^{2}$ The TB epidemic in sub-Saharan Africa is largely driven by the HIV epidemic which disproportionately affects this region. About $70 \%$ of the 36.9 million people living with HIV (PLHIV) globally live in sub-Saharan Africa, of whom $76 \%$ are found in the eastern and southern regions. This is despite the fact that sub-Saharan Africa contributes only $14 \%$ of the world's population. ${ }^{3}$

Of the estimated 10 million people who developed TB disease in 2017, 9\% were PLHIV of whom $72 \%$ lived in the Africa region. ${ }^{4}$ Among those with TB and HIV coinfection, the majority (84\%) were from the Africa region. ${ }^{4}$ Despite this large prevalence, there has been a $44 \%$ decline in the number of HIV/TB associated deaths since 2000. This is largely attributed to an increased uptake of antiretroviral therapy (ART) among those with HIV-associated TB. Coverage of ART in Africa has more than doubled from $35 \%$ in 2005 to $84 \%$ in $2017 .^{45}$ 
ART is associated with a $67 \%$ reduced risk of developing active TB disease among PLHIV ${ }^{6}$ and an even further reduction in recurrent TB. ${ }^{7}$ ART has also been shown to reduce the population incidence of TB by between $27 \%$ and $80 \% .{ }^{89}$ However, despite ART having a TB protective effect at the individual level, which has further been shown to positively correlate with higher updated CD4 cell counts, ${ }^{10} 11$ the incidence of TB still remains higher in the HIV-positive population compared with the HIVnegative population. ${ }^{11}$

To further mitigate TB in this high-risk group, isoniazid preventive therapy (IPT) has also been found to be effective in reducing the incidence of TB and death from TB among PLHIV with further reductions in TB risk of up to $90 \%$ among those receiving both IPT and ART. ${ }^{12-14}$ This has led the WHO to recommend initiation of IPT among PLHIV enrolled in ART care in whom TB has been excluded using a clinical TB screening algorithm. ${ }^{15}$

Zimbabwe is one of the sub-Saharan African countries with a largely HIV-driven TB epidemic and is among the 14 countries globally with a triple burden of TB, TB/ HIV and multidrug resistant TB. ${ }^{4}$ A previous publication from Zimbabwe evaluating a 14-year period from 2000 to 2013 showed that with increasing ART coverage since its inception in the public sector in 2004, annual TB case notification rates more than halved for all forms of TB. Furthermore, the largest declines were among those with recurrent $\mathrm{TB}(53 \%)$, new smear-negative pulmonary TB (58\%) and extrapulmonary TB (58\%). ${ }^{16}$ Zimbabwe also adopted WHO guidance on provision of IPT among PLHIV in 2011. There was an initial pilot phase in 10 health facilities where the delivery of IPT was found to be feasible, and this was followed by nationwide roll-out to most of the public health facilities in the country. ${ }^{17} 18$ The country has also adopted the 'HIV treat all' approach from mid-2016 where those who test HIV positive are immediately eligible for ART initiation regardless of their $\mathrm{CD} 4$ cell count or WHO clinical staging.

We hypothesise that the nationwide scale-up of IPT; the continued increase in ART coverage and the earlier initiation of ART have all had a population impact on reducing $\mathrm{TB}$ incidence in Zimbabwe, translating to further declines in TB case notification rates. Currently, there are no reports or published papers comparing the national scale-up of IPT and ART with national TB case notification rates. We therefore describe in this paper the association between IPT and ART scale-up and annual TB case notification rates in Zimbabwe from 2000 to 2018.

\section{METHODS}

\section{Study design}

This was an ecological study design using aggregate programme data to analyse for trends and associations.

\section{Setting}

Zimbabwe is a developing country in Southern African with a population of 13 million $^{19}$ according to latest census data. There is a gross national index per capita of US $\$ 860^{20}$ and $72.3 \%$ of the population live below the national poverty line. ${ }^{20}$ The country has a high HIV prevalence rate of $15 \%$, and the total number of adults and children living with HIV was estimated at 1.3 million in 2018 according to the Joint United Nations Programme on HIV/AIDS (UNAIDS). ${ }^{21}$

\section{The National Art Programme in Zimbabwe}

Antiretroviral drugs were first offered in public health facilities in 2004 under the National ART Programme at five central level hospitals. As of December 2018, ART was available at $1604(93 \%)$ out of 1722 health facilities nationwide. ${ }^{22}$ Since the inception of the ART programme in 2004, the criteria for initiating ART among adults has shifted from a CD4 cell count threshold of $<200$ cells $/ \mathrm{mL}$ until 2010 , to $<350$ cells $/ \mathrm{mL}$ from 2011 to 2013 and to $<500$ cells $/ \mathrm{mL}$ from 2014 in accordance with WHO guidelines. ${ }^{23-25}$ Those with advanced HIV disease have always been eligible for ART regardless of CD4 cell count.

Towards the end of 2013, there was a shift towards universal eligibility for lifelong ART in Zimbabwe. This started under option B+ when all HIV-infected pregnant and breastfeeding women were initiated on ART irrespective of their $\mathrm{CD} 4$ counts or WHO staging. Over this same period, ART regimens became simpler, less toxic and with a reduced pill burden given the transition to one pill per day fixed-dose combination regimens. From mid-2016 onwards, the country started implementing the 'HIV treat all' approach whereby all patients who were diagnosed HIV positive are immediately eligible for ART initiation regardless of $\mathrm{CD} 4$ cell count or WHO clinical staging.

\section{Intensive TB case finding, IPT initiation and enrolment in art care} All confirmed PLHIV in Zimbabwe are enrolled at ART clinics to receive HIV treatment and care services. According to Zimbabwe's national IPT guidelines, all PLHIV should be screened for active TB at every clinic visit or every encounter with a health worker using the WHO recommended four-symptom TB screening checklist. ${ }^{26}$ If PLHIV present with any of the four symptoms (cough of any duration, night sweats, weight loss and fever), they are considered as presumptive TB cases and have their sputum specimens collected for investigation by smear microscopy or Xpert MTB/Rif assay (Cepheid, Sunnyvale, California, USA) . Those who are negative on smear microscopy or Xpert MTB/Rif assay or both and who have no other symptoms or signs of extrapulmonary or smear-negative PTB, including chest radiography if necessary, are not diagnosed with active $\mathrm{TB}$ and are potentially eligible for IPT.

IPT eligibility has changed over time. At the start of the programme in 2011, all patients enrolled in HIV care were eligible for IPT regardless of ART status, provided active TB was excluded. Since the national ART programme moved towards an 'HIV treat all' approach, all PLHIV in pre-ART care were recalled to health facilities for ART initiation and were prioritised for ART care first and then 
initiated on IPT. All those eligible for IPT are started on a 6-month daily oral dose of isoniazid $(5 \mathrm{mg} / \mathrm{kg}$ body weight for adults or $10 \mathrm{mg} / \mathrm{kg}$ body weight for children) plus a daily low dose of pyridoxine $(25 \mathrm{mg} /$ day). To prevent unnecessary visits to health facilities, resupplies of IPT and pyridoxine are synchronised to ARV resupplies until completion of TB preventive treatment. During clinic visits, patients are assessed through self-reporting and pill counts for adherence, and they are also screened for TB. Patients found to have developed active TB disease are discontinued from IPT and are subsequently treated for TB according to national TB guidelines. ${ }^{26}$ By the end of June 2019, there were $1280(74 \%)$ out of 1722 public health facilities offering IPT in addition to ART across the country's 10 provinces (National ART Programme data).

\section{The National TB Programme in Zimbabwe}

Zimbabwe like other countries with a high prevalence of $\mathrm{TB}$ has a well-established WHO recommended directly observed treatment short course (DOTS) ${ }^{27}$ national TB programme (NTP), whereby TB treatment services are integrated with general health services at all health facilities countrywide. This DOTS model consists of a centralised and prioritised system of TB monitoring, recording and training of TB case management, and there is also a standardised recording and reporting system that allows assessment of treatment results. National TB treatment outcomes are recorded and reported in line with WHO guidelines and are classified as treatment success (cured plus treatment completed), loss to follow-up (LTFU), died, transferred out or not evaluated and failed treatment.

Patients with TB are classified as either new or previously treated TB cases, whereby new cases are those who have never received anti-TB treatment or have previously received anti-TB drugs for $<30$ days, while previously treated TB cases are those patients who have previously received anti-TB drugs for $>1$ month. Prior to 2013, new TB cases were divided into smear-positive pulmonary (PTB), smear-negative PTB or extrapulmonary TB (EPTB) cases, while retreatment patients with TB were categorised as follows: (1) relapse cases, (2) treatment after failure, (3) treatment after LTFU or (4) 'retreatment other' based on smear microscopy. However from 2013 onwards, both new and recurrent TB cases have been classified as new/recurrent bacteriologically confirmed, clinically diagnosed and EPTB cases based on Xpert MTB/Rif results, the preferred first-line TB diagnostic test. The NTP also ensures these drug-susceptible patients with $\mathrm{TB}$ are prescribed a standardised treatment regimen of 6 months for new TB cases or 8 months for recurrent TB cases: doses are observed by a healthcare worker or community health worker for at least the first 2 months of therapy.

\section{Study population}

The study population included: all PLHIV reported nationally in ART care at the end of each year; all PLHIV in ART care who were initiated on IPT annually and all patients annually diagnosed and notified with TB in Zimbabwe between 2000 and 2018.

\section{Patient and public involvement}

Patients or the public were not involved in the design, or conduct, or reporting, or dissemination of our research study.

\section{Data variables and sources of data}

National aggregate data on annual estimated numbers of PLHIV and annual numbers receiving ART were obtained from UNAIDS and WHO. ${ }^{21} 28$ Data on PLHIV estimates are generated annually using the Estimation and Projection Package and Spectrum software ${ }^{29}$ from UNAIDS based on primary collected data from census reports, antenatal clinic surveillance, population-based surveys and programme data. National population figures were obtained from the 2002 and 2012 national census reports with intercensus projections for the periods in between these reports and after 2012. ${ }^{19} 3031$ Data on annual numbers started on IPT were obtained from the Zimbabwe Demographic Health Information System version 2 (DHIS2). Finally, aggregate data on numbers notified each year with TB and further stratified by type and category of TB were obtained from the WHO website. ${ }^{32}$

\section{Analysis and statistics}

Data were analysed by plotting bar and line graphs of the various indicators over time from 2000 to 2018. ART coverage was calculated by dividing the annual total number of PLHIV receiving ART by the annual estimated number of PLHIV, instead of relying on ART coverages reported in annual UNAIDS or WHO reports since these were dependent on changing ART eligibility criteria over the years. IPT coverage rates were calculated as cumulative annual numbers of PLHIV in ART care ever started on IPT divided by annual numbers of PLHIV receiving ART.

Annual TB case notification rates were calculated by dividing annual TB case notifications by annual national population figures to obtain case notifications per 100000 population. Changes in TB notification rates between 2000 and 2018 were determined by comparing the highest TB case notification rates prior to national ART scale-up and the lowest annual TB case notification rates after roll-out of both ART (in 2004) and IPT (in 2012) using tests for proportions in Stata V.15.0 (StataCorp, College Station, Texas, USA). Levels of significance were set at $5 \%$.

\section{RESULTS}

Since the inception of ART in the public sector in 2004, there was a substantial increase in the number and proportion of PLHIV initiated on ART from 8400 $(<1 \%$ ART coverage) in 2004 to over 1 million (88\% ART 


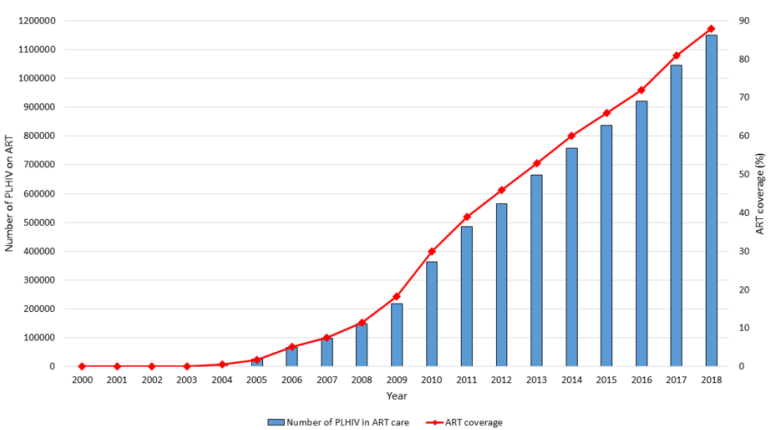

Figure 1 Numbers and coverage of PLHIV alive on ART in Zimbabwe, 2000-2018. ART coverage was calculated using the estimated HIV-infected population as the denominator from national projections obtained from the Joint United Nations Programme on HIV/AIDS Estimation and Projection Package and Spectrum software). ART, antiretroviral therapy; PLHIV, people living with the HIV.

coverage) by the end of 2018 (figure 1). The number and proportion of PLHIV in ART care who were ever started on IPT increased from $98(<1 \%$ coverage $)$ in 2012 to approximately 373917 (33\% coverage) by the end of 2018 (figure 2).

All TB case notification rates increased between 2000 and 2004 from a high of 446 cases/100 000 population to a peak of 509 cases $/ 100000$ population before declining steeply to 315 cases/100 000 population in 2008 (figure 3). Following this, there was a brief increase in case notification rates to 372 cases $/ 100000$ population before a steady decline to an all-time low of 173 cases $/ 100$ 000 population in 2018. A similar trend was observed for new TB cases which constituted the majority of all TB case notifications. Retreatment TB cases increased fourfold between 2001 and 2004 from 13 cases/100 000 population to 58 cases $/ 100000$ population before declining to 20 cases $/ 100000$ population in 2007. Thereafter, case notification rates increased to a plateau of 37 cases/100 000 population in 2009 and 2010 prior to eventually

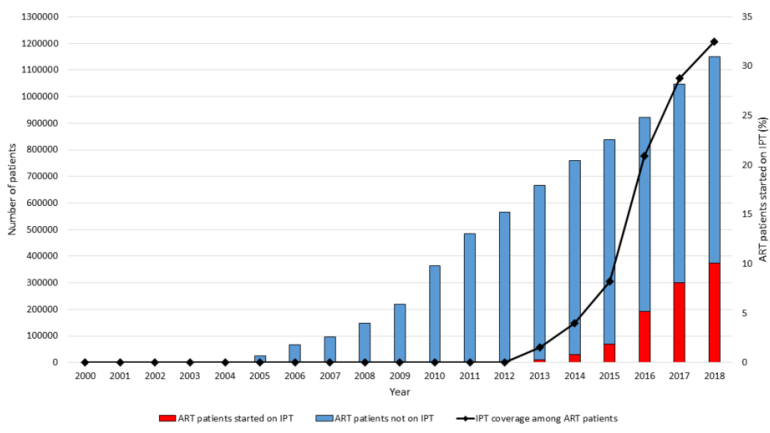

Figure 2 Numbers on IPT and IPT coverage among PLHIV receiving ART in Zimbabwe, 2000-2018. IPT coverage was calculated using the cumulative annual numbers started on IPT as the numerator and cumulative annual numbers alive on ART as reported from the Zimbabwe DHIS2 reporting database. ART, antiretroviral therapy; DHIS, Demographic Health Information System; IPT, isoniazid preventive therapy; PLHIV, people living with the HIV.

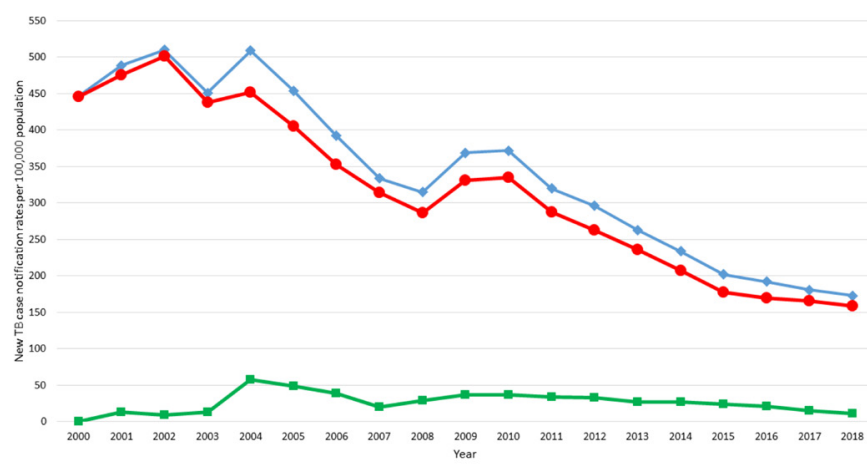

Figure 3 Notification rates of all TB cases in Zimbabwe stratified by TB category, 2000-2018. PTB, pulmonary TB; TB, tuberculosis.

declining steadily over time to 11 cases/100 000 population by the end of 2018 .

Clinically diagnosed PTB cases increased from 242 cases/100 000 population in 2000 to an all-time peak of 284 cases/100 000 population in 2002 and significantly declined although with intermittent increases observed in 2004 and 2009-2010 before reaching an all-time low of 63 cases/ 100000 population in 2017 (figure 4). Bacteriologically confirmed PTB cases also increased from 126 cases/100 000 population in 2000 to an all-time peak of 137 cases / 100000 population in 2002 and later declined steadily to 79 cases/100 000 population in 2008. Thereafter the rate of cases increased slightly before declining to an all-time low of 77 cases/100 000 population in 2018. Extrapulmonary TB case notification rates declined steadily over time from 78 cases $/ 100000$ population in 2000 to their lowest of 16 cases/100 000 population in 2018, with slight increases observed during 2001-2002 and 2008-2009.

In figure 5, there was an observed decline in TB case notifications over the years with increasing ART and IPT coverage since their national roll-out in 2004 and 2012, respectively. In table 1 , there were overall significant declines in all $\mathrm{TB}$ case notification rates between the highest recorded case notification rate prior to ART and IPT national roll-out in comparison with the lowest

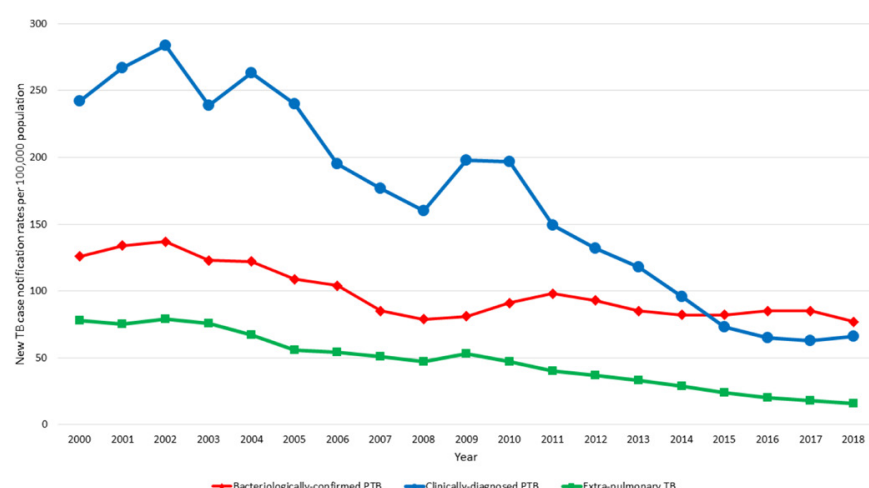

Figure 4 Notification rates of new TB cases in Zimbabwe stratified by type of TB, 2000-2018. PTB, pulmonary tuberculosis; TB, tuberculosis. 


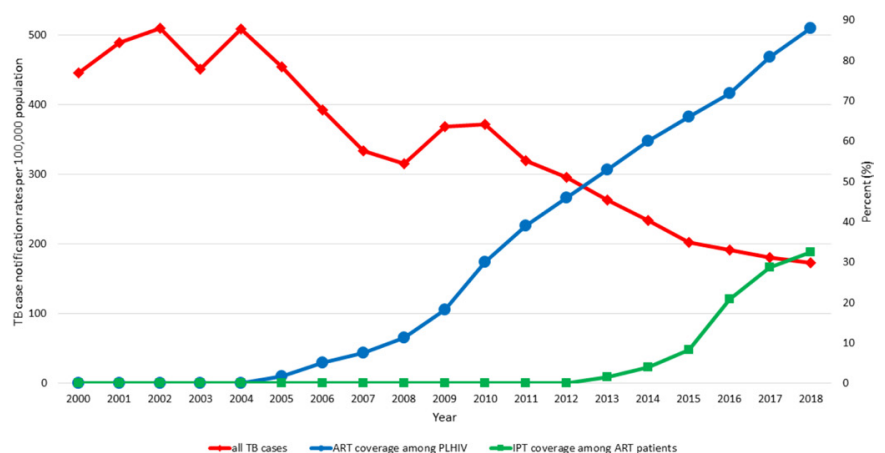

Figure 5 Trends in notification rates of all TB cases versus ART and IPT coverage in Zimbabwe, 2000-2018. (ART coverage was calculated using the estimated HIV-infected population as the denominator from national projections obtained from the UNAIDS Estimation and Projection Package and Spectrum software. IPT coverage was calculated using the cumulative annual numbers started on IPT as the numerator and cumulative annual numbers alive on ART as reported from the Zimbabwe DHIS2 reporting database. ART, antiretroviral therapy; DHIS, Demographic Health Information System; TB, tuberculosis; UNAIDS, United nations Programme for HIV/AIDS.

case notification rates after ART and IPT scale-up (66.1\%, $\mathrm{p}<0.001)$. Similar significant declines in case notification rates were observed among those with new TB (68.3\%, $\mathrm{p}<0.001)$ and among those with new clinically diagnosed PTB $(77.8 \%, \mathrm{p}<0.001)$.

\section{DISCUSSION}

This study is a follow-on of a previous study showing the association between declines in TB case notifications from 2000 to 2013 and scale-up of ART in the public sector since its inception in $2004 .{ }^{16}$ In this current study, we assessed TB case notification rates for a further 5-year period up to 2018 after ART coverage had nearly doubled from nearly half of all estimated PLHIV to nearly 9 in 10 PLHIV and after the National AIDS Programme had attained an IPT coverage of approximately one-third of patients receiving life-saving ART.

Our findings showed that these HIV-related interventions (ART and IPT) were associated with significant declines in TB case notification rates between the highest and lowest reported case notification rates prior to and after the inception of ART and IPT in the public sector, respectively. These declines were observed for all TB cases combined and among those with new $\mathrm{TB}$, in particular those with new clinically diagnosed PTB. In comparison to the previous study, ${ }^{16}$ there was a further percentage decline of TB case notification rates between the lowest recorded $\mathrm{TB}$ case notification rates reported then and those currently presented of $34 \%, 33 \%, 27 \%, 52 \%$ and $59 \%$ for all forms of $\mathrm{TB}$, new $\mathrm{TB}$, new clinically diagnosed PTB, new EPTB and previously-treated TB cases, respectively.

These findings reaffirm the population impact of the pooled TB-protective effect of ART and IPT in a setting where TB is mainly HIV driven. This may also be coupled with implementation of intensified TB case finding among PLHIV to identify those eligible for IPT. Simultaneously, those needing TB treatment can be identified and treated, thus resulting in improved infection control in ART care settings where newly diagnosed TB cases are most prevalent. ${ }^{33} 34$

The further national gains in significantly increasing ART coverage towards the UNAIDS 90\% ART coverage target by adopting an 'HIV treat all' approach in recent years have resulted in $64 \%$ of all estimated PLHIV in Zimbabwe being virally suppressed according to the last 2015-2016 Zimbabwe Population HIV Impact Assessment population-based survey. ${ }^{35}$ This has had a significant impact on the near threefold reduction in the estimated TB incidence in the population from 584 to 221 cases $/ 100000$ population between 2000 and $2017 .^{436}$ In line with our findings, a modelling exercise involving countries from sub-Saharan Africa also showed that the most notable decline in incidence of HIV-associated TB

Table 1 Comparisons of TB case notification rates, stratified by type and category prior to 2004 (at start of ART scale-up) and after ART roll-out and IPT roll-out (2012) in Zimbabwe

\begin{tabular}{|c|c|c|c|c|c|c|}
\hline Type of TB & $\begin{array}{l}\text { Highest } \\
\text { notification rate } \\
\text { (per } 100 \text { 000) }\end{array}$ & Year & $\begin{array}{l}\text { Lowest } \\
\text { notification rate } \\
\text { (per } 100 \text { 000) }\end{array}$ & Year & $\begin{array}{l}\text { Decrease } \\
(\%) \text { between } \\
\text { highest and } \\
\text { lowest }\end{array}$ & P value* \\
\hline All TB & 510 & 2002 & 173 & 2018 & 66.1 & $<0.001$ \\
\hline New TB & 501 & 2002 & 159 & 2018 & 68.3 & $<0.001$ \\
\hline Bacteriologically confirmed PTB & 137 & 2002 & 77 & 2018 & 43.8 & 0.143 \\
\hline Clinically diagnosed PTB & 284 & 2002 & 63 & 2017 & 77.8 & $<0.001$ \\
\hline Extrapulmonary TB & 79 & 2002 & 16 & 2018 & 79.7 & 0.281 \\
\hline Previously treated TB & 58 & 2004 & 11 & 2018 & 81.0 & 0.432 \\
\hline
\end{tabular}

${ }^{*}$ Test of proportions comparing percentage decreases between the highest recorded case notification rate prior to ART and IPT scale-up and the lowest recorded case notification rate after ART roll-out (in 2004) and IPT roll-out (in 2012).

.ART, antiretroviral therapy; EPTB, extrapulmonary TB; IPT, isoniazid preventive therapy; PTB, pulmonary tuberculosis; TB, tuberculosis. 
is seen when those diagnosed HIV positive are immediately initiated on ART compared with delaying ART until 5 years after HIV seroconversion. ${ }^{37}$

Interestingly, the decline in $\mathrm{TB}$ case notifications which was higher in those with clinically diagnosed PTB has been sustained even though TB diagnosis has shifted from smear microscopy to Xpert MTB/Rif. The Xpert MTB/Rif assay is more sensitive in diagnosing TB in patients whose sputum smears are negative for acidfast bacilli (which is more common among PLHIV), ${ }^{38}$ and hence one might have anticipated an increase in numbers of $\mathrm{TB}$ cases detected rather than the observed decrease.

A recent modelling study showed that treatment of latent TB infection can result in a 14-fold decline in global TB incidence between 2013 and 2050 in comparison to no treatment of latent TB infection. ${ }^{39}$ Therefore, despite the observed and encouraging decline in TB case notifications, there are still opportunities for further declines given that IPT coverage is only approximately one-third of PLHIV on ART. Uptake of IPT has slowed down because of resistance of some health workers who fear the development of isoniazid resistance coupled with fears among patients given a few anecdotal reports of serious side effects among some patients taking this prophylactic treatment. Although IPT completion rates in Zimbabwe are higher than reported elsewhere, completion rates are still suboptimal probably due to the increased pill burden of IPT over a 6-9 month duration. ${ }^{17} 18$ Improved completion rates may lead to a further decline in TB case notification rates.

Zimbabwe's planned national roll-out of a shorter regimen of TB preventive therapy (namely, a 12-week course of weekly rifapentine plus isoniazid-3HP) for children and adult PLHIV, following the feasibility phase roll-out in 10 pilot sites beginning in early 2020 , can potentially lead to increased TB preventive therapy coverage. This may lead to further declines in TB incidence among PLHIV in ART care. In comparison to the standard 6-9 months isoniazid monotherapy course, the 3HP prophylactic treatment course has been shown to have higher completion rates and a lower risk of adverse events, particularly hepatotoxicity. ${ }^{40}$

The major strength of this study is that national aggregate data that is routinely tracked as part of HIV/TB global response indicators were used, hence giving a comprehensive picture of what is prevailing in the country over time. The major limitation comes from the ecological design of the study which results in 'the ecological fallacy', whereby other factors that are attributed to the observed decline in TB case notifications over time aside from ART and IPT are not accounted for. ${ }^{41}$ However, our findings are largely reliable as they are coherent with findings from wellconducted studies at the individual level using robust study methodologies. ${ }^{12-14}$ There are other limitations. TB case notifications may underestimate the true TB cases in Zimbabwe over time especially among PLHIV. Despite the use of GeneXpert, the test has been shown to have low sensitivity and specificity in settings were HIV prevalence is $>30 \%{ }^{38}$ There may also have been missed TB cases through suboptimal TB screening among patients attending health facilities and leakages along the care cascade to sputum collection, submission for laboratory diagnosis, return of results and commencement of TB treatment. ${ }^{42-44}$ Nevertheless, based on TB treatment coverages for Zimbabwe that were reported by WHO, these were relatively constant around $70 \%,{ }^{32}$ hence the observed annual TB case notification rates are probably standardised for undiagnosed TB cases.

In conclusion, this study shows continued declining trends of TB case notification rates that support the continued scale-up of ART and TB preventive therapy among PLHIV as key strategies for mitigating the dual burden of HIV/AIDS and TB in Zimbabwe and other regions with HIV-driven TB epidemics.

\section{Author affiliations}

${ }^{1}$ Center for Operational Research, International Union Against Tuberculosis and Lung Disease, Paris, France

${ }^{2}$ AIDS and TB Department, Ministry of Health and Child Care, Harare, Zimbabwe ${ }^{3}$ Department of Clinical Research, Faculty of Infectious and Tropical Diseases, London School of Hygiene and Tropical Medicine, London, UK

${ }^{4}$ World Health Organization Country Office for Zimbabwe, Harare, Harare, Zimbabwe

\section{Twitter Kudakwashe C Takarinda @ktakarinda}

Contributors KCT and ADH conceived and designed the study, and TM-A, CS, RCC, SM, CM and CT read, revised and approved the study protocol. KCT collected the data and analysed them, while TM-A, CS, RCC, SM, CM and CT contributed to interpreting the data. KCT drafted the manuscript, and TM-A, CS, RCC, SM, CM and CT read, critically revised the manuscript for intellectual content and eventually approved the final manuscript.

Funding The authors have not declared a specific grant for this research from any funding agency in the public, commercial or not-for-profit sectors.

Competing interests Technical support was provided through the International Union Against Tuberculosis and Lung Disease (IUATLD). Kudakwashe Takarinda is supported as a Senior Operations Research Fellow from the Centre for Operational Research at the IUATLD, Paris, France.

Patient and public involvement Patients and/or the public were not involved in the design, conduct, reporting or dissemination plans of this research.

Patient consent for publication Not required.

Ethics approval Ethics approval was sought and obtained from the Ministry of Health and Child Care, Harare, Zimbabwe and the Ethics Advisory Group of the International Union Against Tuberculosis and Lung Disease, Paris, France.

Provenance and peer review Not commissioned; externally peer reviewed.

Data availability statement Data are available in a public, open access repository. Data for this study are freely available from the WHO website (https://www.who.int/ tb/country/data/download/en/) for TB data and the UNAIDS website (http://aidsinfo. unaids.org) for ART coverage data. Alternatively data and the statistical code can be shared by the corresponding and lead author upon contacting him on email address kctakarinda@gmail.com.

Open access This is an open access article distributed in accordance with the Creative Commons Attribution Non Commercial (CC BY-NC 4.0) license, which permits others to distribute, remix, adapt, build upon this work non-commercially, and license their derivative works on different terms, provided the original work is properly cited, appropriate credit is given, any changes made indicated, and the use is non-commercial. See: http://creativecommons.org/licenses/by-nc/4.0/.

ORCID iD

Kudakwashe C Takarinda http://orcid.org/0000-0002-2980-7735 


\section{REFERENCES}

1 Dye C, Scheele S, Dolin P, et al. Consensus statement. global burden of tuberculosis: estimated incidence, prevalence, and mortality by country. who global surveillance and monitoring project. JAMA 1999;282:677-86.

2 Pawlowski A, Jansson M, Sköld M, et al. Tuberculosis and HIV coinfection. PLoS Pathog 2012;8:e1002464.

3 United Nations Department of Economic and Social Affairs Population Division (un DESA). World population prospects 2019 data booklet. New York, USA, 2019.

4 World Health Organization. Global tuberculosis report 2018. Geneva, Switzerland: World Health Organisation (WHO), 2018.

5 World Health Organization (WHO). Global tuberculosis control: who report 2010. Geneva, 2010.

6 Lawn SD, Wood R, De Cock KM, et al. Antiretrovirals and isoniazid preventive therapy in the prevention of HIV-associated tuberculosis in settings with limited health-care resources. Lancet Infect Dis 2010;10:489-98.

7 Golub JE, Durovni B, King BS, et al. Recurrent tuberculosis in HIVinfected patients in Rio de Janeiro, Brazil. AIDS 2008;22:2527-33.

8 Miranda A, Morgan M, Jamal L, et al. Impact of antiretroviral therapy on the incidence of tuberculosis: the Brazilian experience, 19952001. PLoS One 2007;2:e826.

9 Middelkoop K, Bekker L-G, Myer L, et al. Antiretroviral therapy and TB notification rates in a high HIV prevalence South African community. J Acquir Immune Defic Syndr 2011;56:263-9.

10 Suthar AB, Lawn SD, del Amo J, et al. Antiretroviral therapy for prevention of tuberculosis in adults with HIV: a systematic review and meta-analysis. PLoS Med 2012;9:e1001270.

11 Gupta A, Wood R, Kaplan R, et al. Tuberculosis incidence rates during 8 years of follow-up of an antiretroviral treatment cohort in South Africa: comparison with rates in the community. PLoS One 2012; 7:e34156-10.

12 Durovni B, Saraceni V, Moulton LH, et al. Effect of improved tuberculosis screening and isoniazid preventive therapy on incidence of tuberculosis and death in patients with HIV in clinics in Rio de Janeiro, Brazil: a stepped wedge, cluster-randomised trial. Lancet Infect Dis 2013;13:852-8.

13 Golub JE, Pronyk P, Mohapi L, et al. Isoniazid preventive therapy, HAART and tuberculosis risk in HIV-infected adults in South Africa: a prospective cohort. AIDS 2009;23:631-6.

14 Golub JE, Saraceni V, Cavalcante SC, et al. The impact of antiretroviral therapy and isoniazid preventive therapy on tuberculosis incidence in HIV-infected patients in Rio de Janeiro, Brazil. AIDS 2007;21:1441-8.

15 World Health Organization (WHO). WHO policy on collaborative TB/HIV activities. guidelines for national programmes and other stakeholders. Geneva: WHO, 2012: 1-34.

16 Takarinda KC, Harries AD, Sandy C, et al. Declining tuberculosis case notification rates with the scale-up of antiretroviral therapy in Zimbabwe. Public Health Action 2016;6:164-8.

17 Takarinda KC, Choto RC, Harries AD, et al. Routine implementation of isoniazid preventive therapy in HIV-infected patients in seven pilot sites in Zimbabwe. Public Health Action 2017;7:55-60.

18 Takarinda KC, Choto RC, Mutasa-Apollo T, et al. Scaling up isoniazid preventive therapy in Zimbabwe: has operational research influenced policy and practice? Public Health Action 2018;8:218-24.

19 Zimbabwe National Statistics Agency (ZIMSTAT). Zimbabwe population census 2012. Harare, 2013.

20 The World Bank. Zimbabwe Data - The World Bank [Internet], 2015. Available: http://data.worldbank.org/country/zimbabwe

21 Joint United Nations Programme for HIV/AIDS (UNAIDS). AIDSinfo [Internet]. Available: http://aidsinfo.unaids.org/

22 Zimbabwe Ministry of Health and Child Care (MOHCC). Global AIDS response progress report 2018: Zimbabwe country report. Harare, Zimbabwe, 2018.
23 World Health Organisation (WHO). Consolidated guidelines on the use of antiretroviral drugs for treating and preventing HIV infection: recommendations for a public health approach June 2013. Geneva: WHO, 2013: 1-269.

24 World Health Organisation (WHO). Antiretroviral therapy for HIV infection in adults and adolescents: recommendations for a public health approach - 2010 revision. 4911. Geneva: WHO, 2010.

25 World Health Organisation (WHO). Antiretroviral therapy for HIV infection in adults and adolescents: Recommendations for a public health approach - 2006 revision. Geneva: WHO, 2006: 1-134.

26 Ministry of Health and Child Care (MOHCC). Zimbabwe TB guidelines. Harare: MOHCC, 2010.

27 World Health Organisation. Treatment of tuberculosis: guidelines for national programmes. Geneva: World Health Organisation (WHO), 1997: 1-77.

28 World Health Organisation (WHO) regional office for Africa. HIV in the who African region: progress towards achieving universal access to priority health sector interventions: 2011 update. Brazzaville, 2011.

29 Ghys PD, Brown T, Grassly NC, et al. The UNAIDS estimation and projection package: a software package to estimate and project national HIV epidemics. Sex Transm Infect 2004;80 Suppl 1:i5-9.

30 ZIMSTAT. Zimbabwe 2002 national census report. Harare, 2004.

31 Zimbabwe National Statistics Agency (ZIMSTAT. 2012 Zimbabwe population census: population projections thematic report. Harare, 2015.

32 World Health Organization. Tuberculosis - Download data as CSV files, 2019.

33 Kranzer K, Houben RM, Glynn JR, et al. Yield of HIV-associated tuberculosis during intensified case finding in resource-limited settings: a systematic review and meta-analysis. Lancet Infect Dis 2010;10:93-102.

34 Martinson NA, Hoffmann CJ, Chaisson RE. Epidemiology of tuberculosis and HIV: recent advances in understanding and responses. Proc Am Thorac Soc 2011;8:288-93.

35 Ministry of Health and Child Care (MOHCC). Zimbabwe populationbased HIV impact assessment (ZIMPHIA) 2015-16: first report. Harare, 2017.

36 World Health Organization. Global tuberculosis control: surveillance, planning, financing WHO report 2002. Geneva, Switzerland, 2002.

37 Williams BG, Granich R, De Cock KM, et al. Antiretroviral therapy for tuberculosis control in nine African countries. Proc Natl Acad Sci U S A 2010;107:19485-9.

38 Walusimbi S, Bwanga F, De Costa A, et al. Meta-Analysis to compare the accuracy of GeneXpert, MODS and the who 2007 algorithm for diagnosis of smear-negative pulmonary tuberculosis. BMC Infect Dis 2013;13:507.

39 Dye C, Glaziou P, Floyd K, et al. Prospects for tuberculosis elimination. Annu Rev Public Health 2013;34:271-86.

40 Hamada Y, Ford N, Schenkel K, et al. Three-Month Weekly rifapentine plus isoniazid for tuberculosis preventive treatment: a systematic review. Int J Tuberc Lung Dis 2018;22:1422-8.

41 Neumark Y. What can ecological studies tell us about death? Isr J Health Policy Res 2017;6:1-5.

42 Kweza PF, Van Schalkwyk C, Abraham N, et al. Estimating the magnitude of pulmonary tuberculosis patients missed by primary health care clinics in South Africa. Int J Tuberc Lung Dis 2018;22:264-72.

43 Takarinda KC, Choto R, Sandy C, et al. How well does the process of screening and diagnosis work for HIV-infected persons identified with presumptive tuberculosis who are attending HIV care and treatment clinics in Harare City, Zimbabwe? Trans $R$ Soc Trop Med Hyg 2018;112:450-7.

44 Mugauri H, Shewade HD, Dlodlo RA, et al. Bacteriologically confirmed pulmonary tuberculosis patients: loss to follow-up, death and delay before treatment initiation in Bulawayo, Zimbabwe from 2012-2016. Int J Infect Dis 2018;76:6-13. 